\title{
Dispersión Legítima De Semillas Por Aves En El Bosque Y Matorral Serrano De La Provincia De Córdoba
}

\author{
Claudia M. Dellafiore, PhD \\ Universidad Nacinal de Río Cuarto - Fac. de Ciencias Exactas, \\ Fco-Qcas y Naturales. Río Cuarto, Córdoba
}

doi: 10.19044/esj.2016.v12n18p56 URL:http://dx.doi.org/10.19044/esj.2016.v12n18p56

\begin{abstract}
Birds play a fundamental role in many ecosystems because through endozoochory they can disperse lots of fruits and herefore its seeds. In the southern hemisphere, temperate forests and hills scrub of Córdoba-Argentina are characterized by great floristic diversity and an important richness of birds. However, only few studies focusing on the role of birds as dispersers of fruits and seeds have been conducted in this ecosystem. The aims of this study was to investigate if birds are dispersing fruits or seeds in the forest and hills scrub; analyse if fruits or seeds dispersion is scattered along seasons; and to assess whether seeds suffer physical damage when passing through the digestive tract of birds. During July, August, September and December 2012, fruits and seeds from birds pellets were collected while walking through two linear transects (length and width) of 1,450 x 20 and $1,200 \times 5 \mathrm{~m}$, respectively. Results showed that birds dispersed seeds during all seasons although the largest proportion was observed during autumn and winter when more than $70 \%$ of birds pellets contained seeds. The highest proportion of damaged seeds occurred in September (11\%), but overall proportion of seeds that had physical damage was very low. Finally, our results revealed that birds have an important role in seeds dispersion on the forest and hills scrub ecosystem, and most of these dispersed seeds were exotic
\end{abstract}

Keywords: Seed dispersal, birds, shrubland anf forest, frugivory, endozoochory

\section{Resumen}

Las aves juegan un rol fundamental en muchos ecosistemas ya que a través de mecanismos como la endozoocoria pueden dispersar una gran variedad de frutos y semillas. En los bosques templados del hemisferio sur 
el bosque y matorral serrano de la provincia de Córdoba-Argentina se caracterizan por presentar una gran diversidad florística y una importante riqueza de aves; sin embargo, son muy escasos los estudios realizados en relación al rol de las aves como dispersoras de frutos y semillas. El objetivo del presente trabajo fue: a) conocer si las aves dispersan frutos y/o semillas en el bosque y matorral serrano, b) conocer que especies de frutos y/o semillas estan siendo dispersadas en las diferentes estaciones del año y c) analizar si las semillas sufren daño físico al pasar por el tracto digestivo de las aves. Para ello se recolectaron fecas frescas sobre dos transectos lineales en las disttintas estaciones del año. Los resultados obtenidos reflejan que las aves dispersan semillas durante todo el año aunque la mayor proporción fue durante el otoño y el invierno cuando mas del $70 \%$ de las muestras contenían semillas. Un muy bajo porcentaje de dichas semillas presentaron daño físico siendo el valor mas elevado en el mes de septiembre donde el $11 \%$ de las mismas estaban dañadas. Concluimos que las aves realizan una “dispersión legítima” de semillas en el ecosistema de bosque y matorral serrano. Las semillas dispersadas fueron tanto de especies exóticas como de especies autóctonas pero con un alto predominio de las primeras.

Palabras clave: dispersión de semillas, aves, bosque y matorral serrano, frugivoría, endozoocoria

\section{Introducción}

En los bosques tropicales las aves son responsables de la dispersión del 90\% de los frutos y semillas presentes (Frankie et al, 1974) y, en muchos casos, la reproducción y reclutamiento exitoso de las plantas dependen de dicha interacción (Thompson, 2006). Entre los bosques templados del hemisferio sur el bosque y matorral serrano de la provincia de Córdoba - Argentina se caracterizan por presentar una gran diversidad florística y una importante riqueza de aves autóctonas (Luti et al., 1979; Nores e Yzurieta, 1983; Nores et al., 1983; Nores, 1996). Entre las plantas presentes exiten especies que producen frutos atractivos para las aves pero se desconoce si dichas aves contribuyen a la “dispersión legítima”de sus semillas. Entendiendo como dispersión legítima la propagación de semillas sin daño físico (Montaldo, 2005). Conocer el rol de las aves como dispersoras de frutos y semillas es de gran importancia ya que la diversidad, funcionamiento e integridad de muchos ecositemas dependen de esta relación mutualista (Armesto et al., 1995; Rozzi et al., 1996; Willson et al., 1996; Aizen et al., 2002).

De acuerdo con lo expuesto anteriormente, el presente trabajo tuvo por objetivos: a) conocer si las aves del bosque y matorral serrano de Córdoba dispersan frutos y/o semillas por endozoocoria, b) conocer que 
especies de frutos y/o semillas estan siendo dispersadas en las diferentes estaciones del año y c) analizar si las semillas sufren daño físico al pasar por el tracto digestivo de las mismas. Este estudio se llevó a cabo bajo la hipótesis general de que las aves actuan como dispersoras legítimas de especies autóctonas y exóticas en el bosque y matorral serrano de la provincia de Córdoba - Argentina.

\section{Materiales y métodos}

El área de estudio comprende una superficie de $3,5 \mathrm{~km}^{2}$ y está ubicada en la ciudad de Alpa Corral, Provincia de Córdoba, Argentina. Dicha área pertenece a la Región Fitogeográfica del Chaco Serrano (Cabrera, 1976). Entre las especies vegetales nativas observadas en el área de estudio se encuentran Lithraea ternifolia, Fagara coco, Celtis ehrenbergiana, Schinus areira, Schinus fasciculatus, Prosopis torquata y varias especies de la familia Poaceae como Stipa sp. y Festuca sp. Además, se encontraron especies exóticas como Ligustrum lucidum, Rubus ulmifolis, Pyracantha atalantoides, Rosa eglanteria y Gleditsia triacanthos.

En el área de estudio se censaron veintiséis especies de aves nativas de América del Sur y al menos dieciséis de ellas consumen frutos y semillas de forma regular (por ej: Saltator aurantiirostris, Poospiza melanoleuca, Coryphospingus cucullatus, Zonotrichia capensis, Turdus rufiventris, entre otras) (Dellafiore obs. pers).

La búsqueda y colecta de fecas se realizó sobre dos transectos lineales de $1.450 * 20 \mathrm{~m}$ y $1.200 * 5 \mathrm{~m}$ durante los meses de julio, agosto, septiembre y diciembre de 2012. Se recolectaron únicamente fecas frescas y debido a que las aves suelen defecar varios pellets en un mismo lugar se consideró como una muestra a todos los pellets recogidos en la misma posición geográfica (GPS). En el laboratorio las fecas fueron pesadas y se realizó el análisis del contenido de las mismas. Para ello fueron desmenuzadas mecánicamente mediante pinza diente de ratón y aguja de disección. El reconocimiento de los ítems fue realizado bajo lupa estereoscópica y las semillas observadas fueron contadas y, cuando fue posible, se identificaron a nivel de especie. Además, dichas semillas fueron analizadas en detalle para registrar posibles daños físico (semillas partidas, tegumento roto, exposición del embrión, deshidratación, etc.).

\section{Resultados}

Se recolectaron 11 muestras de fecas frescas en el mes de junio (75gr), 24 en agosto (48gr), 15 en septiembre (56gr) y 21 en diciembre (40gr). Los resultados obtenidos del análisis de las mismas reflejan que las semillas estuvieron presentes en las fecas de las aves durante todos las estaciones del año aunque la mayor proporción fue observada durante el 
otoño (376 semillas) y el invierno (248 semillas) cuando mas del 70\% de las muestras analizadas contenían semillas (Tabla 1 y Figura 1).

\begin{tabular}{|c|c|c|c|c|c|}
\hline & $\begin{array}{l}\text { Otoño } \\
\text { n=11 } \\
(75 \\
\text { gr) }\end{array}$ & $\begin{array}{c}\text { Invierno } \\
n=24 \\
(48 \text { gr })\end{array}$ & $\begin{array}{c}\text { Primavera } \\
\mathrm{n}=15 \\
(56 \mathrm{gr})\end{array}$ & $\begin{array}{c}\text { Verano } \\
n=21 \\
(40 \mathrm{gr})\end{array}$ & $\begin{array}{l}\text { Total de } \\
\text { semillas }\end{array}$ \\
\hline \multicolumn{6}{|l|}{ Especies Autóctonas } \\
\hline $\begin{array}{c}\text { Celtis ehrenbergiana } \\
\text { Schinus fasciculatus } \\
\text { Poáceas } \\
\text { Solanáceas } \\
\end{array}$ & 26 & $\begin{array}{c}14 \\
2 \\
\end{array}$ & $\begin{array}{l}3 \\
28\end{array}$ & 11 & $\begin{array}{c}3 \\
11 \\
68 \\
2 \\
\end{array}$ \\
\hline \multicolumn{6}{|l|}{ Especies Exóticas } \\
\hline $\begin{array}{l}\text { Ligustrum lucidum } \\
\text { Pyracantha sp } \\
\text { Rubus ulmifolius }\end{array}$ & $\begin{array}{c}275 \\
75\end{array}$ & 232 & 5 & 6 & $\begin{array}{c}512 \\
75 \\
6 \\
\end{array}$ \\
\hline Total semillas & 376 & 248 & 36 & 17 & 677 \\
\hline Total de semillas por gramo & 5 & 5 & 0.6 & 1 & 11.6 \\
\hline
\end{tabular}

Tabla 1: Número de semillas observadas en las fecas de las aves en las diferentes estaciones del año.

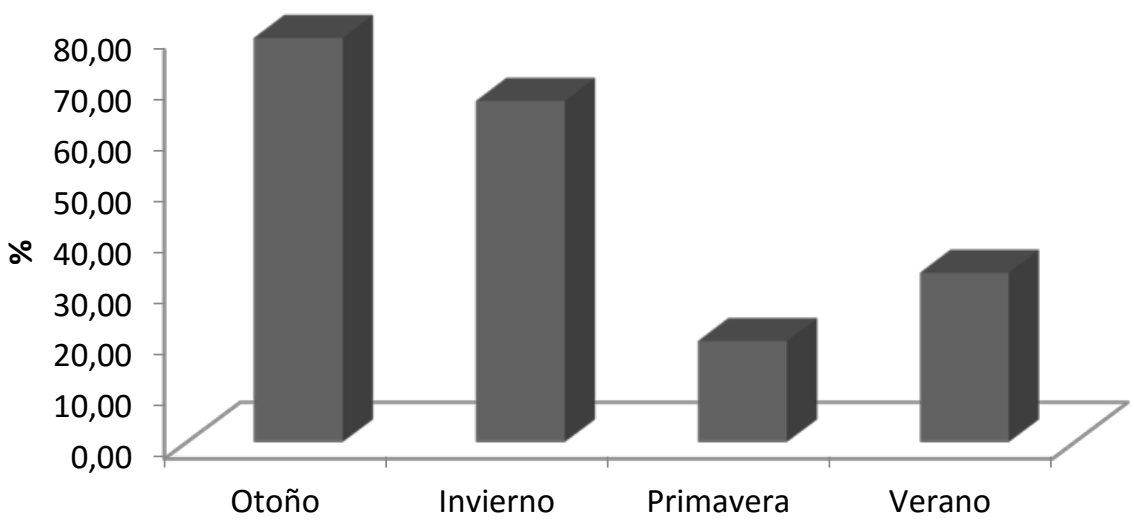

Figura 1: Porcentaje de semillas observadas estacionalmente en las fecas de las aves.

Un muy bajo porcentaje de semillas presentaron daño físico siendo el valor mas elevado en el mes de septiembre donde el $11 \%$ de las semillas estaban dañadas. Durante junio, agosto y diciembre el 99,0\%, 98,9\% y 100\% de las semillas observadas no mostraron daño físico.

Las semillas observadas en las fecas pertenecieron tanto a especies autóctonas como a especies exóticas. Entre las especies autóctonas encontramos Celtis ehrenbergiana, Schinus fasciculatus, poáceas y solanáceas. Entre las semillas de especies exóticas encontramos Ligustrum lucidum, Pyracantha sp. y Rubus ulmifolius (Tabla 1 y Figura 2). 


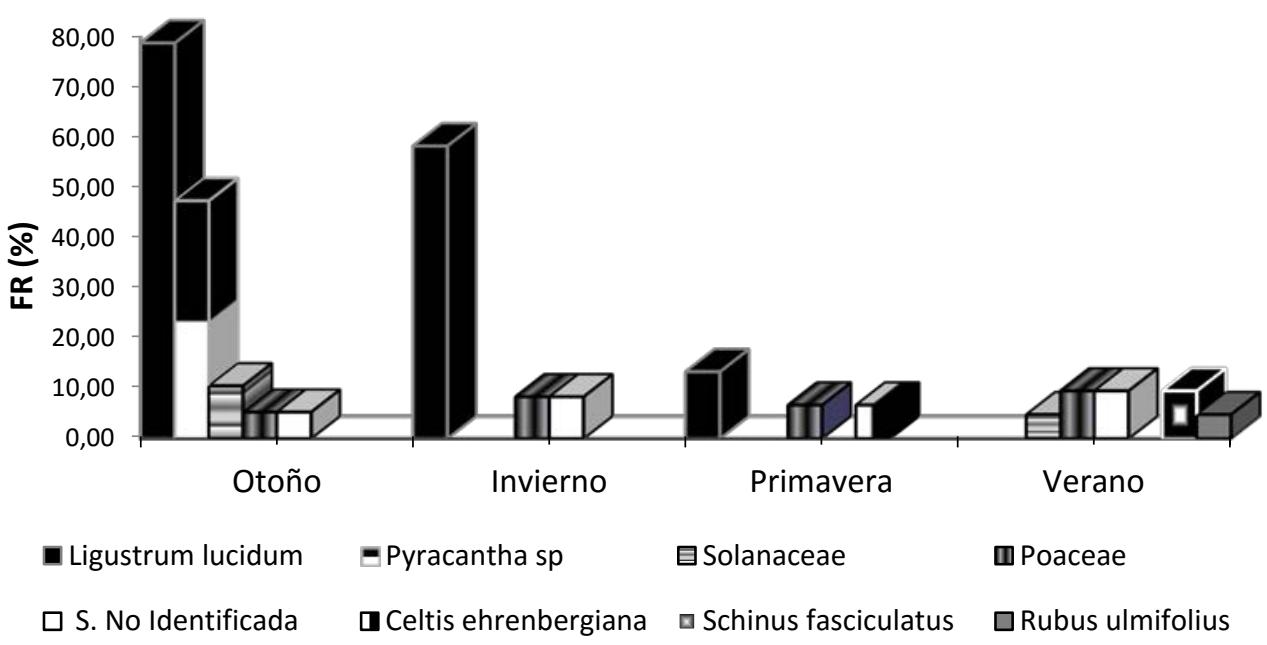

Figura 2: Frecuencia de aparición relativa de semillas en las diferentes estaciones del año.

Las especies autóctonas estuvieron presentes en un bajo porcentaje de las muestras analizadas $\mathrm{y}$, en su mayoría, aparecieron muy ocasionalmente. C.ehrenbergiana solo estuvo presente en primavera (3 semillas), S. fasciculatus en verano (11 semillas) y las solanáceas en invierno ( 2 semillas). Las poáceas estuvieron presentes en otoño, invierno y primavera (26, 14 y 28 semillas respectivamente) y entre el 5 y $9 \%$ de las muestras contenían semillas pertenecientes a esta familia (Tabla 1 y Figuras 2 y 3$)$.

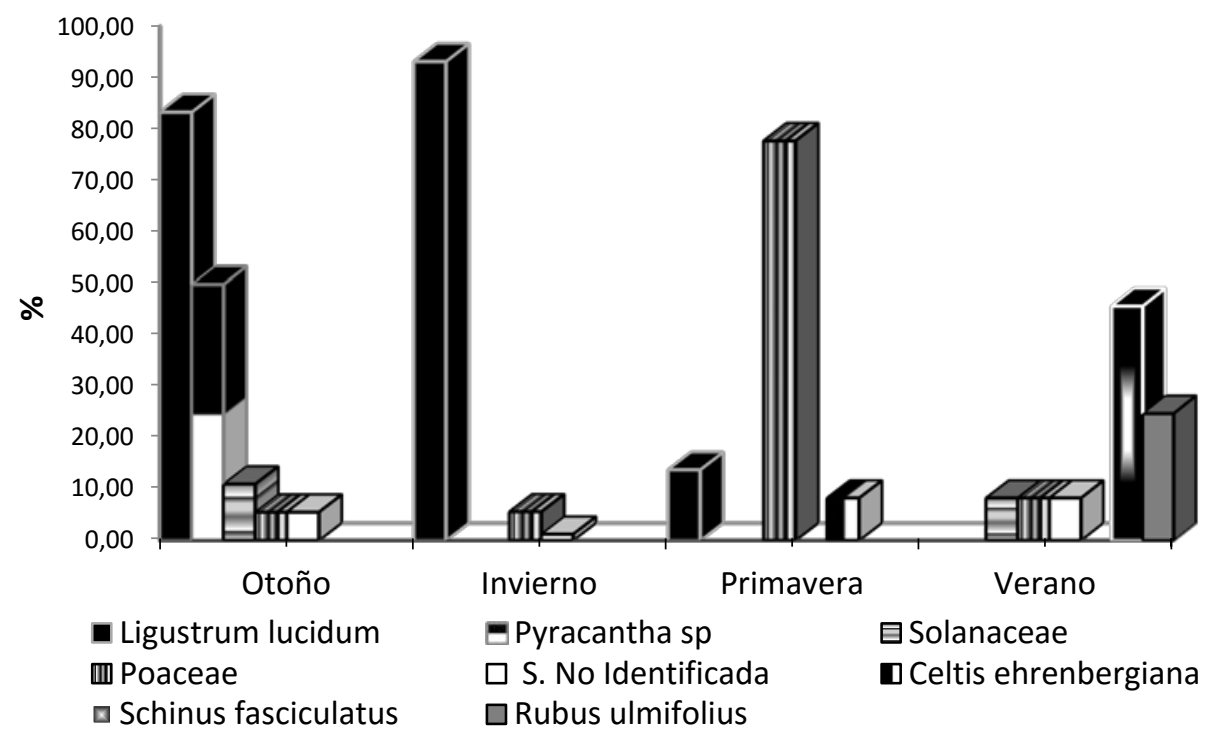

Figura 3: Porcentaje de fecas con las diferentes especies de semillas. 
L. lucidum estuvo presente en la mayoría de las estaciones del año (otoño, invierno y primavera) mientras que Pyracantha sp y R. ulmifolius solo aparecieron en otoño y verano respectivamente (Tabla 1 y Figura 1$). L$. lucidum fue la semillas más abundante con 275 semillas en el otoño y 232 semillas en el invierno y estuvo presente en un elevado porcentaje de muestras (79\% y 58\% respectivamente) y en menor proporción durante el verano donde solo el 13\% de las muestras contenían esta especie (Tabla 1 y Figura 2). Pyracantha sp solo apareció en el otoño pero el $47 \%$ de las muestras contenían esta especie (75 semillas) y R. ulmifolius solo estuvo presente en el verano en un bajo porcentaje de muestras (5\% y 5 semillas) (Tabla 1 y Figuras 2 y3).

\section{Conclusión}

Las aves dispersan importantes cantidades de semillas en el ecosistema de bosque y matorral serrano de la provincia de Córdoba Argentina y la dispersión sería legítima ya que el porcentaje de semillas dañadas fue muy bajo. Las semillas dispersadas fueron tanto de especies exóticas como de especies autóctonas con un alto predominio de las primeras.

Las especies autóctonas encontradas en las fecas de las aves fueron Celtis ehrenbergiana y Schinus fasciculatus las cuales tienen frutos muy atractivos para las aves y existen evidencias de que son una fuente importante de alimento para las mismas (Dellafiore, obs. pers.; Ponce et al., 2012). Sin embargo, el número de semillas observadas fue muy bajo lo cual podría deberse a que las aves consumen la pulpa pero no ingieran las semillas por lo que el aporte a su dispersión sería muy pobre. Es decir, las aves no juegan un papel importante en la dispersión de semillas autóctonas ya que fueron muy pocas las especies encontradas y las semillas dispersadas. A pesar de ello, el hecho de que "una semilla" pueda llegar a sitios alejados de la planta madre sería positivo para las especies involucradas ya que le permitiría incrementar su supervivencia y llegar a nuevas áreas abiertas a la colonización (Janzen 1970; Connell 1971; Augspurger 1983, 1984; Augspurger and Kelly 1984; Clark \& Clark 1984; Howe et al., 1985, Herrera, 2002).

Con relación a las especies exóticas se pudo observar que las aves dispersan cantidades importantes de Pyracantha sp y Ligustrum lucidum (Tabla 1 y Figura 2) y, como las semillas no sufren daños físicos al pasar a través del tracto digestivo, juegan un rol muy importante en la propagación de dichas especies. Además, las semillas de Pyracantha sp no pierden su viabilidad (Dellafiore et. al, 2015) por lo que podrán germinar inmediatamente o pasar a formar parte del banco de semillas hasta encontrar las condiciones favorables para su germinación. En el caso de Ligustrum 
lucidum mayores estudios son necesarios para conocer la viabilidad de las semillas luego de atravesar el tracto digestivo de las aves. Por otro lado, Rubus ulmifolius apareció en bajas cantidades en las fecas pero ellas no pierden su viabilidad (Traveset et al., 2001) por lo que las aves contribuirían a su dispersión y supervivencia como ya se mencionó previamente para el caso de las especies autóctonas.

Las especies exóticas - Pyracantha sp y Ligustrum lucidum dispersadas por las aves poseen una alta producción de frutos los cuales están disponibles durante gran parte del año. De acuerdo con nuestros resultados, dichos frutos forman parte de la dieta de las aves durante el otoño y el invierno cuando la disponibilidad de alimento es más escasa en nuestra área de estudio. Por lo tanto, cabe esperar que esta mayor disponibilidad de alimento en épocas de escases mejore el "fitness" de las aves que aprovechan este recurso. Esto a su vez podría promover cambios en las comunidades de aves presentes en el bosque y matorral serrano. Mayores estudios son necesarios para conocer como las especies exóticas impactan sobre las comunidades de aves y las consecuencias de erradicar dichas especies una vez que se han establecido en el ecosistema y han pasado a formar parte de la dieta de las aves frugívoras.

\section{Agradecimientos}

Agradecemos a la Secretaría de Ciencia y Técnica de la Universidad Nacional de Río Cuarto por al apoyo financiero y a los familiares y amigos que ayudaron con las tareas de campo.

\section{References:}

Aizen, M.A., Vázquez, D.P. \& Smith-Ramirez, C. (2002). Historia natural y conservación de los mutualismos planta-animal del bosque templado de Sudamérica austral. Revista Chilena Historia Natural 75:79-97.

Armesto, J.J., \& Gutiérrez, J.R. (1980). Aplicación de algunas técnicas de muestreo en el análisis de la vegetación de Chile central. Archivos de Biología y Medicina Experimentales 13:403-414.

Augspurger, C. K. (1983). Seed dispersal by the tropical tree Platypodium elegans, and the escape of its seedlings firom fungal pathogens. Journal Ecology, 71:759-771.

Augspurger, C. K. (1984). Seedling survival among tropical tree species: interactions of dispersal distance, light- gaps, and pathogens. Ecology 65:1705-1712.

Augspurger, C. K., \& Kelly, C. K. (1984). Pathogen mortality of tropical tree seedlings: experimental studies of the effects of dispersal distance, seedling density, and light conditions. Oecologia (Berl.) 61:211-217. 
Cabrera, A.L. (1976). Regiones fitogeográficas Argentinas. 2ed. Enciclopedia Argentina Agrícola y Jardinería. ACME, Buenos Aires. 85p. Clark, D. A \& Clark, D. B. (1984). Spacing dynamics of a tropical rain forest tree: evaluation of the Janzen-Connell model. The American Naturalist, 124:769-788.

Connell, J. H. (1971). On the role of natural enemies in preventing competitive exclusion in some marine animals and in rain forest trees. Pages 298-310 in P. J. den Boer and G. Gradwell, eds. Dynamics of populations. Proceedings of the Advanced Study Institute on dynamics of numbers in populations, Oosterbeek, The Netherlands, September 7-18, 1970. Center for Agricultural Publishing and Documentation, Wageningen Dellafiore, C.M., Rosa M.J., \& Scilingo, V. (2015). ¿Afectan las aves la germinación del arbusto Pyracantha atalantoides (Rosaceae)?. Research Journal of the Costa Rica Distance Education University 7 (2): 295-299.

Frankie, G.W., Baker, H.G. , \& Opler, P.A. (1974). Comparative phenological studies of tree in tropical wet and dry forests in the lowlands of Costa Rica. Journal of Ecology 62: 881-919.

Herrea, C.M. (2002) Seed dispersal by vertebrates. In: Herrera CM y Pellmyr O. Plant - Animal Interactions. An Evolutionay Approach. Blackwell Science, 313 pp.

Howe, H. F., Schupp, E. W. \& Westley, G. C. (1985). Early consequences of seed dispersal for a Neotropical tree (Virola surinamensis). Ecology, 66:781-791.

Janzen, D. H. (1970). Herbivores and the number of tree species in tropical forests. The American Naturalist, 104:501- 528.

Luti, R., Beltrán, A., Ferreyra, N., Galera, M., Berzal, M., Nores, M., Herrera, M. \& Barrera, J.C. (1979) Vegetación. Pp. 267- 368 In: Vázquez, J., Miatello, R. \& Roque, M. (eds) Geografía física de la Provincia de Córdoba. Editorial Bold, Buenos Aires.

Montaldo, N.H. (2005). Aves frugívoras de un relicto de selva subtropical ribereña en

Argentina: manipulación de frutos y destino de las semillas. El Hornero 20 (2): 163-172.

Nores, M., \& Yzurieta, D. (1983). Nuevas localidades para aves Argentinas. Parte V. Historia Natural 3: 159-160.

Nores, M., Yzurieta, D. \& Miatello, R. (1983). Lista y distribución de las aves de Córdoba, Argentina. Boletín Academia Nacional de Ciencias, Córdoba 56: 1-114.

Nores, M. (1996). Avifauna de la provincia de Córdoba. Pp. 255- 337. In: Di Tada, I. E. \& Bucher, E. (eds.) Biodiversidad de la provincia de Córdoba. Vol. I. Fauna. Univ. Nac. Río Cuarto, Córdoba 
Ponce, A.M., Grilli, G. \& Galetto, L. (2012). Frugivoría y remoción de frutos ornitócoros en fragmentos del bosque chaqueño de Córdoba (Argentina). Bosque 33(1):33-41.

Rozzi, R., Martinez, D., Willson, M. F. \& Sabag, C. (1996). Avifauna de los Bosques Templados de Sudamérica. Pp.135-152 In: Armesto, J.J., Villagrán, C. \& Arroyo, M.T.K. (eds). Ecología de los bosques nativos de Chile. Editorial Universitaria, Santiago de Chile.

Traveset, A., Riera, N. \& Mas, R.E. (2001). Passage through bird guts causes interspecific differences in seed germination characteristics. Functional Ecology, 15:669-675.

Thompson. JN. (2006). Mutualistic webs of species. Science, 312: 372 373.

Willson, M.F., Smith-Ramirez, C., Sabag, C. \& Hernandez, J.H. (1996). Mutualismos entre plantas y animales en bosques templados de Chile. Pp. 251-264 In: Armesto, J.J., Villagrán, C. \& Arroyo, M.T.K. (eds). Ecología de los bosques nativos de Chile. Editorial Universitaria. Santiago de Chile, Chile. 